\title{
Activation and involvement of the lateral-posterior nucleus of the thalamus after a single generalized tonic-clonic seizure
}

\author{
Eliza Yumi de Freitas Sonoda a,*, Roberta Monterazzo Cysneiros ${ }^{\text {b }}$, Ricardo Mario Arida ${ }^{\text {c }}$, \\ Esper Abrão Cavalheiro ${ }^{a}$, Fulvio Alexandre Scorza ${ }^{a}$ \\ a Departamento de Neurologia Experimental, Universidade Federal de São Paulo/Escola Paulista de Medicina (UNIFESP/EPM), São Paulo, Brazil \\ b Programa de Pós-graduação em Distúrbios do Desenvolvimento, Laboratório de Neurobiologia, Universidade Presbiteriana Mackenzie, São Paulo, Brazil \\ c Departamento de Fisiologia, Universidade Federal de São Paulo/Escola Paulista de Medicina (UNIFESP/EPM), São Paulo, Brazil
}

\section{A R T I C L E I N F O}

\section{Article history}

Received 28 November 2012

Revised 19 March 2013

Accepted 23 March 2013

Available online 18 May 2013

\section{Keywords:}

Neuronal activation

Cell excitability

Pilocarpine

SUDEP

Neuronal loss

\begin{abstract}
A B S T R A C T
The lateral-posterior thalamic nuclei (LP) have been shown to play an important role in controlling epileptic activity. In addition, thalamic atrophy and neuronal loss have been observed in epilepsy. The objective of this study was to investigate whether lateral-posterior neuronal activation may be observed shortly after a single generalized seizure in rats submitted to the pilocarpine model of epilepsy. The results showed an increased lateral-posterior activation as soon as the seizure occurred, suggesting that neuronal loss in the thalamus is not only the consequence of chronic epilepsy.
\end{abstract}

(C) 2013 Elsevier Inc. Open access under the Elsevier OA license

\section{Introduction}

Epilepsy is the most common serious neurological condition, and its relationship with specific cerebral structures is crucial, particularly the thalamic complex. As evidence for this, an experimental study investigating the interictal cerebral metabolic rate by 14C-2DG autoradiography in a chronic model of epilepsy clearly demonstrated an increase in glucose utilization by several brain regions [1]. The most relevant finding was a consistent rise of the metabolic rate in the lateral-posterior thalamic nuclei (LP), suggesting that the LP may be involved in the cerebral circuitry controlling epileptic activity during interictal intervals [1]. Then, the contribution and the participation of LP on spontaneous recurrent seizure (SRS) activity in the same epilepsy model were demonstrated [2]. Briefly, it was shown that bilateral lateral-posterior lesions by ibotenic acid in rats with epilepsy resulted in a five-time increase in seizure frequency, suggesting that the lateral-posterior thalamic nucleus is one of the most important thalamic nuclei involved in the inhibition of spreading mechanisms [2]. Along these lines, these data clearly demonstrate the possible role of the LP in the inhibitory control of excessive activation during a seizure. Following this line of reasoning, it is pertinent to think that the integrity of this nucleus may be critical for decreasing the probability of sudden unexpected death in epilepsy (SUDEP)

\footnotetext{
* Corresponding author at: Rua Botucatu 862, Edifício Leal Prado, Vila Clementino, Postal Code (CEP): 04023-900, São Paulo, SP, Brazil. Fax: +55 1155739304. E-mail address: elizasonoda@gmail.com (E.Y.F. Sonoda).
}

occurrence, since high seizure frequency is one of the main risk factors for SUDEP [3].

The lateral-posterior nucleus is important not only for the regulation of brain hyperexcitation but also for the control of cardiac autonomic function. The thalamus receives inputs from the insular cortex and amygdala, which are interconnected with each other and with autonomic nuclei of the brain stem and spinal cord. Additionally, its projections to the hypothalamus, periaqueductal gray, and nucleus ambiguus give rise to parallel pathways that influence both parasympathetic and sympathetic outflows associated with cardiac and respiratory control [4]. Previous findings in the literature have associated abnormal activity of thalamic nuclei with nocturnal apneic episodes [5] as well as with cardiac arrhythmias [6] and altered heart rate variability [7]. Recent studies performed in our laboratory have found transient alterations in heart rate and heart rate variability (such as decreased RR intervals, SDNN, and RMSSD) in LP-injured rats free from epilepsy [8]. Animals submitted to the pilocarpine model of epilepsy also showed decreased RR intervals, and RMSSD and increased LF\% [8]. It has been recognized that seizures can be associated with heart rate changes and ECG abnormalities [9], and cardiac arrhythmias associated with respiratory changes may also be an important cause or factor for the occurrence of SUDEP [10]. In our previous study [8], morphological analyses of the lateral-posterior nucleus in rats with epilepsy were also undertaken and showed a $62.05 \%$ reduction in the immunohistochemistry for Neu-N (100\% being the staining quantified in the control group). There were no significant differences for Nissl staining, although qualitative differences in the 
shape of the stained cells were observed between the group with epilepsy and the control group [8].

However, a question that has not yet been addressed is whether alterations in the lateral-posterior nucleus may be observed in the early stages of epileptogenesis (e.g., after the first seizure) or whether lateral-posterior abnormalities require longer periods to be observed (e.g., after the occurrence of many seizures). Therefore, the objective of the present study was to investigate whether the lateral-posterior neuronal activation may be observed shortly after a single generalized tonic-clonic seizure in rats submitted to the pilocarpine model of epilepsy.

\section{Methods}

Adult male Wistar rats $(\mathrm{n}=15)$ were divided randomly into three groups. The first group was composed of rats that received pilocarpine $(\mathrm{n}=05)$. Status epilepticus (SE) was induced according to the procedure described previously [11,12]. In brief, 30 min after methylscopolamine injection ( $1 \mathrm{mg} / \mathrm{kg} \mathrm{sc}$, Sigma, USA, used to reduce the peripheral effects of pilocarpine), pilocarpine $(450 \mathrm{mg} / \mathrm{kg}$ ip, Sigma, USA) was administered to rats. Only rats that displayed a tonic-clonic seizure (classified according to the Racine's seizure index [13]) were included in this study and perfused as soon as this tonic-clonic seizure occurred. The second group was composed of rats that were placed in $\mathrm{CO}_{2}$ chamber and then perfused as soon as unconscious $(n=05)$. This group is extremely important because the lateral-posterior nucleus can be activated because of apnea associated with a seizure, and hence, this group controls for the apnea factor. The third group was composed of control rats. These rats were injected with saline solution and perfused after $30 \min (n=05)$. After the perfusion process, the brains were fixed, sliced $40 \mu \mathrm{m}$ in vibratome (Leica $\left.{ }^{\circledR}\right)$, and processed for c-Fos immunohistochemistry. The procedures involving the animals and their care at the
Experimental Neurology Laboratory of the Federal University of São Paulo (CEP Protocol 0401/11) conformed with the institution's guidelines, which comply with the International Ethical Guidelines for Biomedical Research [14].

A Nikon E600FN microscope equipped with a digital camera was used for image digitalization. Quantification of c-fos immunolabeled cells was performed using the ImageJ software (National Institutes of Health, Bethesda, MD, USA) by an individual blinded to the treatments. Following background subtraction, the threshold was determined so that all labeled nuclei could be recognized. Quantitative analysis was performed on three sections from every animal (the mean count for each animal was used in the statistical analysis). The appropriate areas were identified according to the atlas of Paxinos and Watson [15]. Cell densities were obtained by dividing the total number of positive cell nuclei by the area of the region examined at $20 \times$ magnification. The amount of c-fos immunolabeled cells (expressed as the average number of cells per $\mathrm{mm}^{2}$ ) was compared between groups by means of a Kruskal-Wallis one-way analysis of variance (with a Mann Whitney $U$ test for individual comparisons).

\section{Results}

Fig. 1 shows representative images of c-Fos expression from control, $\mathrm{CO}_{2}$ chamber, and experimental rats. Qualitative analysis in c-Fos expression showed that the lateral-posterior nucleus is not active in the control condition. On the other hand, the generalized seizure induced by pilocarpine injection activated the lateral-posterior nucleus after a few minutes from the tonic-clonic seizure initiation. The apnea caused by the $\mathrm{CO}_{2}$ chamber did not induce increased activation in this nucleus, indicating that the activation of lateralposterior neurons was caused by the seizure per se (see the Discussion section for details). Fig. 2 shows box plots representing the quantification of c-fos expression for each group. The Kruskal-

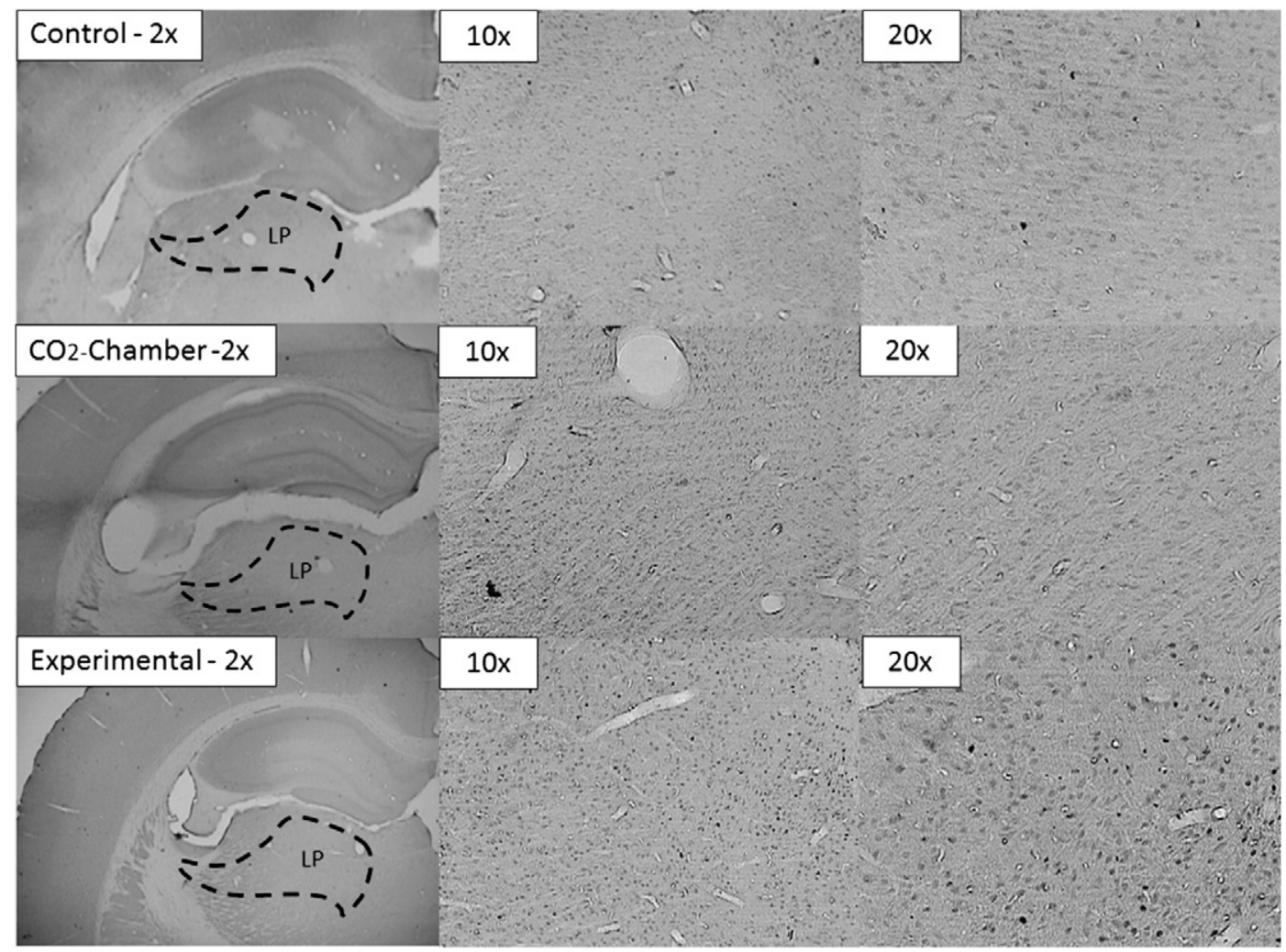

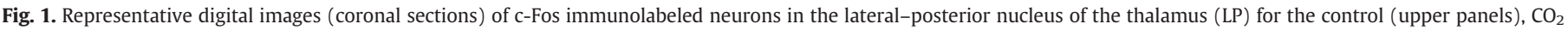

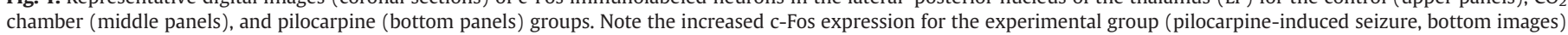
as compared with the control and $\mathrm{CO}_{2}$ chamber groups. 


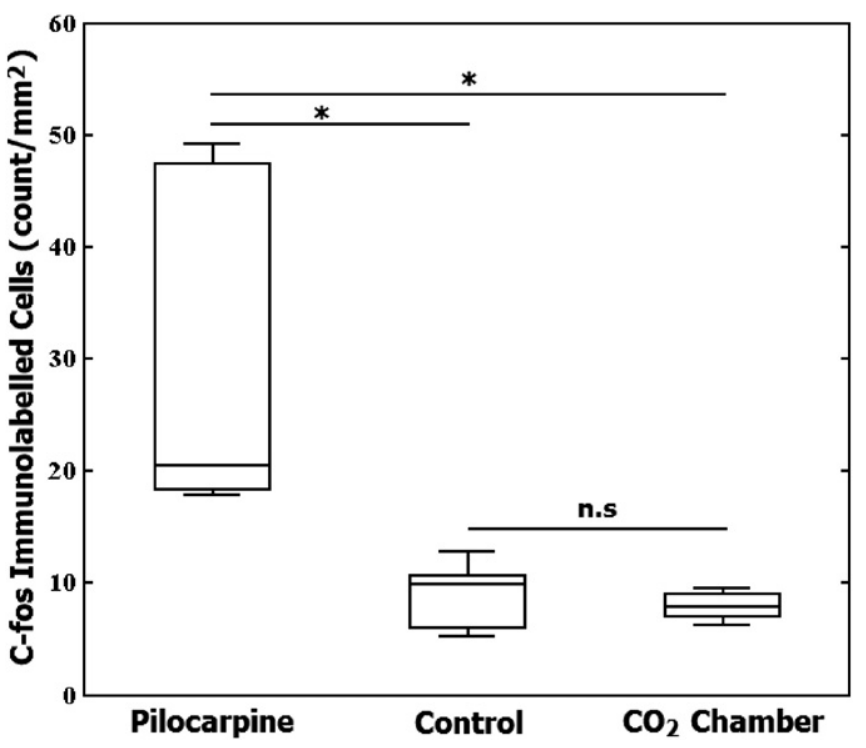

Fig. 2. Density of c-fos immunolabeled neurons in the lateral-posterior nucleus of the thalamus. Box plots for the pilocarpine, control, and $\mathrm{CO}_{2}$ chamber groups are shown from left to right. Horizontal lines in the box plots represent the median, upper, and lower quartiles; the whiskers plot the range. Asterisk $\left({ }^{*}\right)$ denotes significant differences $(\mathrm{P}<0.05)$ between groups. n.s. $=$ not significant.

Wallis test identified significant differences between groups $\left(\mathrm{H}_{(2)}=9.5, \mathrm{P}=0.0087\right.$ ). Pairwise comparisons (Mann Whitney $U$ tests) showed higher density of c-fos immunolabeled cells for the pilocarpine group as compared with the control $(\mathrm{P}=0.009)$ and the $\mathrm{CO}_{2}$ chamber $(\mathrm{P}=0.009)$ group. No significant differences were found between the control and $\mathrm{CO}_{2}$ chamber groups $(\mathrm{P}=0.602)$.

\section{Discussion}

Besides its role in controlling heart rate and heart rate variability, the lateral-posterior nucleus is very important because of the variety of ways it controls the spread of limbic seizures [1] and because of its crucial role in the initiation and/or generalization of cerebral circuitry involved with controlling spontaneous recurrent seizures [2]. The present research adds to the current knowledge by showing that the increased lateral-posterior activation is observed shortly after the first seizure in the life of the animal. The use of the $\mathrm{CO}_{2}$ chamber group was very important to control for the possible effect of apnea (associated with seizure) in increasing the activation of the LP. In average, the time it took for the animals administered with $\mathrm{CO}_{2}$ to be rendered unconscious was approximately $2 \mathrm{~min}$, which is longer than the seizure period of the pilocarpine animals (i.e., the pilocarpine animals were perfused as soon as the tonic-clonic seizure occurred). Therefore, the increased c-fos expression observed in the pilocarpine group (with no changes in c-fos expression for the $\mathrm{CO}_{2}$ chamber group) indicates that the increased lateral-posterior activation was not due to apnea but probably due to a mechanism inherent to the seizure per se.

In epileptic seizures, the thalamus undergoes permanent changes while participating in seizure generalization. Furthermore, thalamic atrophy and neuronal loss have been observed in animal models of temporal lobe epilepsy [16]. Patients who died after SE presented neuronal loss in the thalamus ipsilateral to the seizure focus [17]. In the present study, we found increased activation of the lateral-posterior nucleus as soon as the first seizure occurred, suggesting that thalamic damage may be present at the early stages of epileptogenesis, which may contribute to trigger the mechanisms responsible for chronic epilepsy and SUDEP. However, we still do not know whether this increased activation is reversible or whether it can become more robust and irreversible with the persistence of chronic epilepsy.
Neuronal activation in the lateral-posterior nucleus can lead to alterations in the cardiovascular autonomic nervous system and may be a risk factor for the occurrence of SUDEP. Therefore, we should focus on the importance of the integrity of the thalamic complex in epilepsy, especially the lateral-posterior nucleus, due to its function on SRS initiation and/or generalization and its relationship with heart rate and heart rate variability [8].

The increased activation of the LP observed by c-Fos immunohistochemistry in this study suggests that high levels of excitation may trigger an excitotoxicity mechanism, thereby leading to neuronal death and decreased thalamic volume. Decreased thalamic volume is usually observed in both cerebral sides $[18,19]$ and is not related to epilepsy duration because abnormal thalamic volume was also observed in children newly diagnosed with epilepsy [20,21]. Therefore, the mechanisms behind neuronal death and decreased volume in the thalamus must be further investigated so as to provide the scientific basis for the development of therapeutic interventions for preventing SRSs and SUDEP. Mapping the levels of thalamic activation through different stages of epilepsy models is the first step, and requires further studies.

Another interesting question to be investigated is the potential use of deep brain stimulation in the LP. For instance, electrical stimulation of the anterior nucleus of the thalamus has been shown to be effective in the treatment of patients with drug-refractory partial and secondarily generalized seizures [22]. However, clinical evidence has proceeded with only limited understanding of the basic mechanisms of brain stimulation, and research studies with animal models often lead to contradictory results, with some pointing to antiseizure effects [23], while others suggest no effect [24] or even a proseizure effect [25]. Such contradictory outcomes are likely associated with details in stimulation parameters, sites of stimulation, and other unknown factors [26]. The acute lateral-posterior activation found in the present study highlights the important role of the lateral-posterior thalamic nucleus in controlling the spread of limbic and recurrent seizures and its potential association with the mechanisms underlying SUDEP. Therefore, future investigations on the use of LP deep brain stimulation to control seizures and prevent SUDEP should be considered.

We can conclude that the neuronal loss in the thalamus may not be only the consequence of chronic epilepsy as we observed that neuronal activation may also occur shortly after the first and only generalized seizure. This neuronal activation is probably a consequence of an attempt to control the seizure. However, hyperexcitability may lead to vicious circle: the more neurons are activated, the more neurons die, and other healthy neurons are activated. Therefore, it is suggested that thalamic damage may begin at the early stages of epileptogenesis, which may contribute to trigger the mechanisms responsible for chronic epilepsy and SUDEP. The probable mechanisms of thalamic activation that can lead to neuronal death in epilepsy should be investigated.

\section{Acknowledgments}

The authors thank FAPESP, CInAPCe-FAPESP, FAPESP/CNPq/ MCT - Instituto Nacional de Neurociência Translacional, and CNPq for supporting this study.

\section{References}

[1] Scorza FA, Sanabria ER, Calderazzo L, Cavalheiro EA. Glucose utilization during interictal intervals in an epilepsy model induced by pilocarpine: a qualitative study. Epilepsia 1998;39:1041-5.

[2] Scorza FA, Arida RM, Priel M, Calderazzo L, Cavalheiro EA. The contribution of the lateral posterior and anteroventral thalamic nuclei on spontaneous recurrent seizures in the pilocarpine model of epilepsy. Arq Neuropsiquiatr 2002;60:572-5.

[3] Nilsson L, Farahmand BY, Persson PG, Thiblin I, Tomson T. Risk factors for sudden unexpected death in epilepsy: a case-control study. Lancet 1999;353:888-93.

[4] Britton JW, Benarroch E. Seizures and syncope: anatomic basis and diagnostic considerations. Clin Auton Res 2006;16:18-28. 
[5] Santarnecchi E, Sicilia I, Richiardi J, Vatti G, Polizzotto NR, Marino D, et al. Altered cortical and subcortical local coherence in obstructive sleep apnoea: a functional magnetic resonance imaging study. J Sleep Res 2012.

[6] Mameli P, Mameli O, Tolu E, Padua G, Giraudi D, Caria MA, et al. Neurogenic myocardial arrhythmias in experimental focal epilepsy. Epilepsia 1988;29:74-82.

[7] Massimini M, Porta A, Mariotti M, Malliani A, Montano N. Heart rate variability is encoded in the spontaneous discharge of thalamic somatosensory neurones in cat. J Physiol 2000;526(Pt 2):387-96.

[8] Sonoda EY. Morphological and functional analysis of the thalamic lateral posterior nucleus in an experimental model of epilepsy: possible role in sudden unexpected death in epilepsy (in Portuguese). Universidade Federal de São Paulo; 2011 [Unpublished Ph.D. thesis].

[9] Yuen AW, Sander JW. Is omega-3 fatty acid deficiency a factor contributing to refractory seizures and SUDEP? A hypothesis. Seizure 2004;13:104-7.

[10] So EL. Demystifying sudden unexplained death in epilepsy-are we close? Epilepsia 2006;47(Suppl. 1):87-92.

[11] Scorza FA, Arida RM, Naffah-Mazzacoratti Mda G, Scerni DA, Calderazzo L, Cavalheiro EA. The pilocarpine model of epilepsy: what have we learned? An Acad Bras Cienc 2009;81:345-65.

[12] Turski WA, Cavalheiro EA, Schwarz M, Czuczwar SJ, Kleinrok Z, Turski L. Limbic seizures produced by pilocarpine in rats: behavioural, electroencephalographic and neuropathological study. Behav Brain Res 1983:9:315-35.

[13] Racine RJ, Gartner JG, Burnham WM. Epileptiform activity and neural plasticity in limbic structures. Brain Res 1972;47:262-8.

[14] Howard-Jones N. A CIOMS ethical code for animal experimentation. WHO Chron 1985;39:51-6.

[15] Paxinos G, Watson C. The rat brain in stereotaxic coordinates. 2nd ed. Sydney; Orlando: Academic Press; 1986.

[16] Bertram EH, Mangan PS, Zhang D, Scott CA, Williamson JM. The midline thalamus: alterations and a potential role in limbic epilepsy. Epilepsia 2001;42:967-78.
[17] Mori H, Mizutani T, Yoshimura M, Yamanouchi H, Shimada H. Unilateral brain damage after prolonged hemiconvulsions in the elderly associated with theophylline administration. J Neurol Neurosurg Psychiatry 1992;55:466-9.

[18] Keller SS, Wieshmann UC, Mackay CE, Denby CE, Webb J, Roberts N. Voxel based morphometry of grey matter abnormalities in patients with medically intractable temporal lobe epilepsy: effects of side of seizure onset and epilepsy duration. J Neurol Neurosurg Psychiatry 2002;73:648-55.

[19] Seidenberg M, Hermann B, Pulsipher D, Morton J, Parrish J, Geary E, et al. Thalamic atrophy and cognition in unilateral temporal lobe epilepsy. J Int Neuropsychol Soc 2008;14:384-93.

[20] Cormack F, Gadian DG, Vargha-Khadem F, Cross JH, Connelly A, Baldeweg T. Extrahippocampal grey matter density abnormalities in paediatric mesial temporal sclerosis. Neuroimage 2005;27:635-43.

[21] Pulsipher DT, Seidenberg M, Guidotti L, Tuchscherer VN, Morton J, Sheth RD, et al. Thalamofrontal circuitry and executive dysfunction in recent-onset juvenile myoclonic epilepsy. Epilepsia 2009;50:1210-9.

[22] Fisher R, Salanova V, Witt T, Worth R, Henry T, Gross R, et al. Electrical stimulation of the anterior nucleus of thalamus for treatment of refractory epilepsy. Epilepsia 2010;51:899-908.

[23] Takebayashi S, Hashizume K, Tanaka T, Hodozuka A. Anti-convulsant effect of electrical stimulation and lesioning of the anterior thalamic nucleus on kainic acid-induced focal limbic seizure in rats. Epilepsy Res 2007:74:163-70.

[24] Ziai WC, Sherman DL, Bhardwaj A, Zhang N, Keyl PM, Mirski MA. Target-specific catecholamine elevation induced by anticonvulsant thalamic deep brain stimulation. Epilepsia 2005;46:878-88.

[25] Lado FA. Chronic bilateral stimulation of the anterior thalamus of kainate-treated rats increases seizure frequency. Epilepsia 2006;47:27-32.

[26] Mirski MA, Rossell LA, Terry JB, Fisher RS. Anticonvulsant effect of anterior thalamic high frequency electrical stimulation in the rat. Epilepsy Res 1997;28: 89-100. 